\title{
Spin-Orbit Engineering of Semiconductor Heterostructures
}

\author{
Federico Bottegoni $^{a}$, Henri-Jean Drouhin ${ }^{a} *$ Guy Fishman $^{b}$, and Jean-Eric Wegrowe ${ }^{a}$ \\ a Ecole Polytechnique, LSI, CNRS and CEA/DSM/IRAMIS, Palaiseau, F-91128 and \\ ${ }^{b}$ Univ Paris-Sud, IEF, CNRS, Orsay, F-91405
}

(Dated: November 17, 2018)

\begin{abstract}
We present a systematic construction of the probability-current operator, based on a momentum power expansion of effective Hamiltonians. The result is valid in the presence of a Rashba term and when a D'yakonov-Perel contribution is included. We propose practical tools for spin-orbit engineering of semiconductor heterostructures. We apply this formalism to a paradigmatic system, the interface between two semi-infinite media, on one side a free-electron-like material and on the other side a barrier material with spin-orbit interaction. We show that the usual boundary conditions, namely the continuity of the envelope function and of a velocity at the interface, according to the BenDaniel-Duke approach, comply with the conservation of the probability current only when first- (Rashba-like) and second-order (free-electron-like) terms are taken into account in the Hamiltonian. We revisit the boundary conditions and we prove that the envelope function may be discontinuous at the interface.
\end{abstract}

PACS numbers: 72.25.Dc, 71.70.Ej, 73.40.Gk 


\section{INTRODUCTION}

The probability current is a fundamental concept in quantum mechanics, which connects the wave-like description of a quasi-particle to the notion of transport current. When we consider a general Schrödinger problem where the Hamiltonian is

$$
\widehat{H}_{0}=\frac{\widehat{\mathbf{p}}^{2}}{2 m}+\mathfrak{U}(\mathbf{r})
$$

where the real potential $\mathfrak{U}(\mathbf{r})$ is periodic in a crystalline solid and $m$ is the free-electron mass, we are led to the usual definition of free-electron current probability: ${ }^{1}$

$$
\mathbf{J}^{f}[\psi]=\operatorname{Re}\left[\psi^{*} \frac{\hat{\mathbf{p}}}{m} \psi\right]=\frac{\hbar}{m} \operatorname{Im}\left[\psi^{*} \nabla \psi\right] .
$$

However, in condensed-matter systems in the presence of Spin-Orbit Interaction (SOI), the potential is no longer real so that a redefinition of this quantity is mandatory. A debated example of this subtle point is provided by semiconductors-based systems, whose proper treatment requires consideration of the Hamiltonian

$$
\widehat{H}=\widehat{H}_{0}+\widehat{H}_{S O}
$$

with

$$
\widehat{H}_{S O}=\frac{\hbar}{4 m^{2} c^{2}}(\nabla \mathfrak{U} \times \hat{\mathbf{p}}) \cdot \hat{\sigma} .
$$

Following the arguments developed by Hoai Nguyen et al.,$\frac{2}{2}$ it is reasonable to express the full Hamiltonian, involving SOI terms, as an effective Hamiltonian which consists of momentum-operator $\hat{\mathbf{p}}$-power series expansion: Indeed, beside the kinetic energy, quadratic in $\hat{\mathbf{p}}$, the SOI provides leading terms that are linear and cubic in $\hat{\mathbf{p}}$, known respectively as Rashba ${ }^{3}$ and D'yakonov-Perel (DP) ${ }^{4}$ terms. Then, since the SOI potential is non real, a more general definition of the probability current $\mathbf{J}[\psi]$ has necessarily to be taken into account. Considering interactions that include higher-order polynomial terms in the Hamiltonian, we have to deal with an effective Hamiltonian of order $n$.

Furthermore, an open question, strictly linked to the one above, concerns the definition of spin current (SC). Indeed, in semiconductor physics, that provides paradigmatic systems for spintronics, it is known that the SC standard definition, used by many authors, $\underline{5}-\underline{10}$ can be suitably applied to two dimensional (2D) systems with Rashba SOI, but fails to describe spin-dependent transport phenomena in bulk cubic semiconductors, where SOI induces a 
DP term in the conduction band. The existence of extra-current terms was pointed out in Ref. 11. Drouhin et al. $\frac{12}{}$ have shown that a redefinition of $\mathrm{SC}$ is mandatory to obtain a unified treatment, enlightening the fact that a properly-symmetrized spin-current operator $\hat{\mathbf{J}}_{\uparrow(\downarrow)}[\psi]$, where $\uparrow(\downarrow)$ refers to up (down) spin channel, gives unexpected results when applied to tunneling through evanescent states in GaAs barriers.

As pointed out by Rashba in Ref. 5, there are still concerns relying on the fact that a consistent theory of spin transport currents has not been formulated yet. From a general point of view, it means that we cannot immediately approach such a topic in terms of non equilibrium thermodynamics. In fact, a difficulty relies on the definition of system in order to formulate relevant balance equations and also on the boundary terms which should possibly be included in the effective Hamiltonian. Recently, Shi et al. $\underline{13}$ have proposed an alternative spin-current operator, satisfying the continuity equation, that allegedly supports important conclusions concerning conservation of spin currents, $\underline{14} \underline{-16}$ but which appears to rely on non-explicit assumptions (see Sec. II).

The inclusion of SOI in the Hamiltonian of a system has direct and practical consequences in heterostructures, where a consistent analysis of the tunneling phenomena is required. The pragmatic BenDaniel-Duke (BDD) approach, $\frac{17}{7}$ that perfectly works when dealing with quadratic Hamiltonians under effective-mass approximation, cannot be straightforwardly extended because it is not always possible to ensure both the continuity of the envelope function and the conservation of the probability current, which is mandatory under steadystate conditions. Then it is necessary to revisit both the probability-current expression and the boundary conditions. This is in line with the ideas of Harrison. 18

In this paper, we present a systematic construction of the probability-current operator $\hat{\mathbf{J}}$, based on an effective Hamiltonian written as a $\hat{\mathbf{p}}$-power series expansion. We show the relation between the velocity operator and the current operator, evidencing the simple structure of the extra terms. This yields easy and compact calculations whereas explicit treatments in particular cases resulted in lengthy calculations. $\underline{11}$ The current operator can be subsequently used to build the SC operators according to the procedure described in Ref. 12. Then, we introduce proper matching conditions at the boundaries, which generalize the BDD procedure, the simplest efficient way to deal with semiconductor heterostructures. Finally, we illustrate our method on three examples: the case of a quadratic Hamiltonian, where we recover the usual situation (continuity of the envelope function and of the velocity), 
the case where a Rashba term is added as a perturbation to the BDD Hamiltonian (there we find that the envelope function is continuous, but its derivative is discontinuous), and the case where a cubic DP term is added to the BDD Hamiltonian (where we prove that the envelope function cannot be continuous).

The layout of this paper is as follows: Sec. II, we give a general construction of current operators and a derivation of local properties. Sec. III, we introduce a general Hamiltonian $\hat{H}^{(n)}$ as a $n^{t h}$-degree homogenous function of momentum-operator coordinates; we consistently derive the velocity operator and we show that a proper symmetrization yields the Hermitian current operator $\widehat{\mathbf{J}}$. Sec. IV, we propose boundary conditions which are suitable to deal with heterostructures. Sec. V, we apply our method to electron tunneling through a [110]-oriented GaAs barrier. Sec. VI, we show how to extend the construction procedure to the spin current operators.

\section{GENERAL DEFINITION OF CURRENT OPERATORS}

A difficulty, that arises when a current operator is taken into account, relies on the correct definition of the system and of its boundaries: in fact, considering the density $\rho$ of a physical quantity, we need to satisfy the continuity equation of $\mathbf{J}$, defining a source term $G$, so that:

$$
\frac{\partial \rho}{\partial t}+\nabla \cdot \mathbf{J}=G
$$

As pointed out by Shi et al. in Ref. 13, the continuity of $\mathbf{J}$ can be ensured by introducing a general source term $G$, as in Eq. 5, but the source term is not uniquely defined and this leads to possible confusion when considering the conservation laws in terms of nonequilibrium thermodynamic equations. $\underline{\underline{19}}$ In any case, we first need to state clearly the local properties of a current operator, postponing the analysis of its global properties. For this purpose, we consider a linear operator $\widehat{A}$ that does not explicitly depend on time and acts over a generic state $\psi$. In the following we adopt the notation $(\widehat{A})=(\psi \mid \widehat{A} \psi)=\psi^{\dagger} \widehat{A} \psi$ used in Ref. 2. The general Schrödinger problem reads:

$$
i \hbar \frac{\partial}{\partial t} \psi=\widehat{\mathcal{H}} \psi
$$

where $\widehat{\mathcal{H}}$ may be any Hamiltonian. For example $\widehat{\mathcal{H}}$ may be equal to $\widehat{H}$ (defined in Eq. 3 ) or to $\widehat{H}_{\text {eff }}$ (defined below in Eq. 26). We explicitly develop the derivative of $\widehat{A}$ with respect 
to time:

$$
\frac{\partial}{\partial t}(\widehat{A})=\frac{\partial}{\partial t}\left(\psi^{\dagger} \widehat{A} \psi\right)=\frac{\partial}{\partial t}\left(\psi^{\dagger}\right) \widehat{A} \psi+\psi^{\dagger} \widehat{A}\left(\frac{\partial}{\partial t} \psi\right)
$$

and with the help of Eq. 6] we obtain:

$$
\frac{\partial}{\partial t}(\widehat{A})=-\frac{1}{i \hbar}(\widehat{\mathcal{H}} \psi)^{\dagger} \widehat{A} \psi+\frac{1}{i \hbar} \psi^{\dagger} \widehat{A}(\widehat{\mathcal{H}} \psi)=\frac{1}{i \hbar}\left[\psi^{\dagger} \widehat{A} \widehat{\mathcal{H}} \psi-(\widehat{\mathcal{H}} \psi)^{\dagger} \widehat{A} \psi\right]
$$

If $\widehat{A}$ is an Hermitian matrix (the elements of which are complex numbers, not differential operators)

$$
(\widehat{\mathcal{H}} \psi)^{\dagger} \widehat{A} \psi=\left(\psi^{\dagger} \widehat{A} \widehat{\mathcal{H}} \psi\right)^{*}
$$

so that we can rewrite Eq. 8 in a more suitable way that we refer to as the local form of Ehrenfest theorem:

$$
\frac{\partial}{\partial t}(\widehat{A})=\frac{2}{\hbar} \operatorname{Im}\left(\psi^{\dagger} \widehat{A} \widehat{\mathcal{H}} \psi\right)
$$

The integration over the whole space leads to the well known Ehrenfest's theorem, whose global form is valid for any (possibly differential) Hermitian operator $\widehat{A}$ :

$$
\frac{\partial}{\partial t}\langle\widehat{A}\rangle=\frac{1}{i \hbar}[\langle\psi|\widehat{A} \widehat{\mathcal{H}}| \psi\rangle-\langle\widehat{\mathcal{H}} \psi|\widehat{A}| \psi\rangle]=\frac{1}{i \hbar}\langle\psi|[\widehat{A}, \widehat{\mathcal{H}}]| \psi\rangle
$$

We can write

$$
\frac{\partial}{\partial t}(\widehat{A})=\frac{1}{\hbar} \operatorname{Im}\left(\psi^{\dagger}\{\widehat{A}, \widehat{\mathcal{H}}\} \psi\right)+\frac{1}{\hbar} \operatorname{Im}\left(\psi^{\dagger}[\widehat{A}, \widehat{\mathcal{H}}] \psi\right)
$$

with $\{\widehat{a}, \widehat{b}\}=\widehat{a} \widehat{b}+\widehat{b} \widehat{a}$, and, by integration over the whole space, we get

$$
\int \mathrm{d}^{3} r \operatorname{Im}\left(\psi^{\dagger}\{\widehat{A}, \widehat{\mathcal{H}}\} \psi\right)=0 .
$$

The time derivative of $(\widehat{A})$ is composed of two parts, concerning two different physical processes: we respectively recognize in Eq. 12 the divergence of the current and the source term $G$ associated to $\widehat{A}$

$$
\nabla \cdot \mathbf{J}_{A}=-\frac{1}{\hbar} \operatorname{Im}\left(\psi^{\dagger}\{\widehat{A}, \widehat{\mathcal{H}}\} \psi\right)=-\frac{1}{\hbar} \operatorname{Im}\left(\psi^{\dagger}\{\widehat{A}, \widehat{\mathcal{H}}-\mathcal{U}\} \psi\right)
$$

where any real potential $\mathcal{U}$ vanishes when taking the imaginary part of the anticommutator, and

$$
G=\frac{1}{\hbar} \operatorname{Im}\left(\psi^{\dagger}[\widehat{A}, \widehat{\mathcal{H}}] \psi\right)
$$


The above procedure has two advantages: first, we have expressed in a general form all the quantities entering Eq. 5 through commutators and anticommutators; then we have related the probability-current expression directly to the local properties of its corresponding operator, without taking into account a closed system (such a procedure does not automatically imply that the integral of $\nabla \cdot \mathbf{J}_{A}$ over the crystal only is zero). The choice of considering open systems makes the current operator involve Dirac distributions to deal properly with possible discontinuities at the boundaries of a subsystem. It has to be noted that it is always possible to include the source $G$ term in the form of a current $\mathbf{J}_{G}, G=\nabla \cdot \mathbf{J}_{G}$ so that the conservation equation becomes

$$
\frac{\partial}{\partial t}(\widehat{A})+\nabla \cdot\left(\mathbf{J}_{A}-\mathbf{J}_{G}\right)=\frac{\partial}{\partial t}(\widehat{A})+\nabla \cdot \mathcal{J}=0
$$

where $\mathcal{J}=\mathbf{J}_{A}-\mathbf{J}_{G}$ is divergence-free in steady-state regime. For instance, if we look for $\mathbf{J}_{\mathrm{G}}=\nabla U_{G}$, the potential $U_{G}$ is a solution of the Laplacian problem $\Delta U_{G}=G$. Moreover, adding to $\mathbf{J}_{G}$ the term $\nabla \times \mathcal{A}_{G}$, where $\mathcal{A}_{G}$ is an arbitrary vector field, does not affect the conservation equation. At this stage, the boundary conditions are not under control. Shi et al. $\underline{\underline{13}}$ observe that it might often happen that

$$
\int_{\mathbb{V}} \mathrm{d}^{3} r G=0
$$

where the integration is performed over the volume of the system $(\mathbb{V})$. Then

$$
\int_{\mathbb{V}} \mathrm{d}^{3} r G=\int_{\mathbb{V}} \mathrm{d}^{3} r \nabla \cdot \mathbf{J}_{G}=\int_{\mathbb{S}} \mathbf{J}_{G} \cdot \mathrm{d} \mathbf{s}=0
$$

where the volume integral is changed into a surface integral through Ostrogradski's theorem (here $\mathbb{S}$ is the surface limiting $\mathbb{V}$ and ds is the surface element along the normal to $\mathbb{S}$ ). Such a relation is obviously satisfied provided that $\mathbf{J}_{G} \cdot \mathrm{d} \mathbf{s}=0$, i.e., provided that $\mathbf{J}_{G}$ is a tangential vector to $\mathbb{S}$, which is "physically" reasonable. Shi et al. further assume that $\mathbf{J}_{\mathbf{G}}$ "is a material property that should vanish outside the sample": this is a more restrictive and questionable hypothesis. For instance in the case of a magnetic field, the effect of the associated vector potential cannot a priori be overlooked outside the sample. Anyway, let us assume that $\mathbf{J}_{\mathbf{G}}=\mathbf{0}$ at the surface $\mathbb{S}$. Following the calculation by Shi et al., it is straightforward to show, after partial integration where the boundary contribution cancels, that

$$
\int \mathrm{d} y \mathrm{~d} z \mathrm{~d} x x\left(\frac{\partial J_{G, x}}{\partial x}+\frac{\partial J_{G, y}}{\partial y}+\frac{\partial J_{G, z}}{\partial z}\right)=-\int \mathrm{d}^{3} r J_{G, x}
$$


where $J_{G, x}, J_{G, y}$, and $J_{G, z}$ are the Cartesian components of $\mathbf{J}_{\mathbf{G}}$. Then

$$
\begin{aligned}
\int \mathrm{d}^{3} r \mathbf{J}_{G} & =-\int \mathrm{d}^{3} r \mathbf{r} \nabla \cdot \mathbf{J}_{G}=-\int \mathrm{d}^{3} r \mathbf{r} G \\
& =-\frac{1}{\hbar} \int \mathrm{d}^{3} r \mathbf{r} \operatorname{Im}\left(\psi^{\dagger}[\widehat{A}, \widehat{\mathcal{H}}] \psi\right)=-\frac{1}{\hbar} \int \mathrm{d}^{3} r \operatorname{Im}\left(\psi^{\dagger} \mathbf{r}[\widehat{A}, \widehat{\mathcal{H}}] \psi\right) .
\end{aligned}
$$

It is easy to check that, provided that $[\widehat{A}, \mathbf{r}]=0$,

$$
\mathbf{r}[\widehat{A}, \widehat{\mathcal{H}}]=[\widehat{A} \mathbf{r}, \widehat{\mathcal{H}}]-i \hbar \widehat{v} \widehat{A}
$$

where $[\mathbf{r}, \widehat{\mathcal{H}}]=i \hbar \widehat{v}$. Thus

$$
\begin{aligned}
\int \mathrm{d}^{3} r \mathbf{J}_{G} & =-\frac{1}{\hbar} \int \mathrm{d}^{3} r \operatorname{Im}\left(\psi^{\dagger}[\widehat{A} \mathbf{r}, \widehat{\mathcal{H}}] \psi\right)+\int \mathrm{d}^{3} r \operatorname{Re}\left(\psi^{\dagger} \widehat{v} \widehat{A} \psi\right) \\
& =-\frac{1}{\hbar} \int \mathrm{d}^{3} r \operatorname{Im}\left(\psi^{\dagger}[\widehat{A} \mathbf{r}, \widehat{\mathcal{H}}] \psi\right)+\int \mathrm{d}^{3} r \widetilde{\mathbf{J}}_{A} .
\end{aligned}
$$

Here, $\widetilde{\mathbf{J}}_{A}$ is the canonical current defined as

$$
\widetilde{\mathbf{J}}_{A}=\operatorname{Re}\left(\psi^{\dagger} \widehat{v} \widehat{A} \psi\right)=\psi^{\dagger} \frac{\widehat{v} \widehat{A}+\widehat{A} \widehat{v}}{2} \psi .
$$

According to Eq. 8, we can write

$$
\int \mathrm{d}^{3} r \mathbf{J}_{G}=-\int \mathrm{d}^{3} r\left[\frac{\mathrm{d}(\widehat{A} \mathbf{r})}{\mathrm{d} t}-\widetilde{\mathbf{J}}_{A}\right]=\int \mathrm{d}^{3} r\left[\widetilde{\mathbf{J}}_{A}-\frac{\mathrm{d}(\widehat{A} \mathbf{r})}{\mathrm{d} t}\right] .
$$

Shi et al. define the effective current density as $\overline{\mathbf{J}}_{G}$

$$
\overline{\mathbf{J}}_{G}=\widetilde{\mathbf{J}}_{A}-\frac{\mathrm{d}(\widehat{A} \mathbf{r})}{\mathrm{d} t} .
$$

We have the two following relations which define respectively the total current $\mathcal{J}$ and the effective total current $\overline{\mathcal{J}}$

$$
\begin{aligned}
& \mathcal{J}=\mathbf{J}_{A}-\mathbf{J}_{G}, \\
& \overline{\mathcal{J}}=\mathbf{J}_{A}-\overline{\mathbf{J}}_{G}=\frac{\mathrm{d}(\widehat{A} \mathbf{r})}{\mathrm{d} t}+\left(\mathbf{J}_{A}-\widetilde{\mathbf{J}}_{A}\right) .
\end{aligned}
$$

Provided $\mathbf{J}_{A}-\widetilde{\mathbf{J}}_{A}=0$, i.e. when making the confusion between the canonical and the true currents (which is justified only for Hamiltonians up to second order in $\widehat{\mathbf{p}}$, see Sec. III), the effective total current becomes $\overline{\mathcal{J}}=\mathrm{d}(\widehat{A} \mathbf{r}) / \mathrm{d} t$, which is Eq. 5 in the papers by 
Shi et al. $\frac{13}{n}$ and also by Zhang et al., $\frac{15}{}$ and is the cornerstone of their further calculations. After a careful analysis, this relation appears to be derived under very special conditions so that it cannot be general. Moreover, the meaning of the so-called effective currents and their relationship with the true currents are not clear. Their use to tackle local transport equations is not justified.

\section{PROBABILITY CURRENT OF AN EFFECTIVE HAMILTONIAN}

\section{A. Formulation of the general $n^{\text {th }}$-order Hamiltonian}

Considering effective Hamiltonians, we deal with general expressions given by momentum series expansions, i.e., constructed from the energy expressed as wave-vector-component series expansion after the substitution $\{k \longrightarrow-i \nabla\}$. We write the effective Hamiltonian $\widehat{H}_{e f f}$ as follows:

$$
\widehat{H}_{e f f}=\widehat{H}_{\mathbf{p}}+V(\mathbf{r})
$$

where $V(\mathbf{r})$ is a potential which may be the potential of a single barrier or the one of a superlattice, for example, $\widehat{H}_{\mathbf{p}}$ is such that

$$
\widehat{H}_{\mathbf{p}}=\sum_{n} \sum_{\substack{l(k) \in\{x, y, z\} \\ k=1, \ldots, n}} c_{l(1), l(2), \cdots, l(n)} \widehat{p}_{l(1)} \ldots \widehat{p}_{l(n)}=\sum_{n} \widehat{H}^{(n)}
$$

where $\widehat{p}_{l(k)}$ is the momentum operator associated to the $l(k)$ Cartesian coordinate and where $c_{l(1)}, \ldots, l(n)$ are Hermitian matrices which are invariant under permutation of the subscripts. The abstract form of Eq. 27] allows us to perform easy calculations. In Sec. IIIB we show how to handle such a general expression to deal with concrete situations.

Formally, we perform the identification

$$
\underbrace{c_{x} \ldots c_{x}}_{\alpha} \underbrace{c_{y} \ldots c_{y}}_{\beta} \underbrace{c_{z} \ldots c_{z}}_{\gamma}=c^{x \ldots x}, \underbrace{y \ldots y}_{\beta}, \underbrace{z \ldots z}_{\gamma}
$$

where $\alpha, \beta$, and $\gamma$ are integers. We obtain

$$
\widehat{H}^{(n)}=\left(c_{x} \widehat{p}_{x}+c_{y} \widehat{p}_{y}+c_{z} \widehat{p}_{z}\right)^{n} .
$$

Given Eqs. 27, 28, and 29, let us note that only terms such as $c_{x x}$ or $c_{x y}$ (for $n=2$ ) are meaningful, a term such as $c_{x}$ being only a trick in the calculation. 
Alternatively, one can write

$$
\widehat{H}^{(n)}=\sum_{\alpha+\beta+\gamma=n} c^{\alpha \beta \gamma} \widehat{p}_{x}^{\alpha} \widehat{p}_{y}^{\beta} \widehat{p}_{z}^{\gamma}
$$

with

$$
c^{\alpha \beta \gamma}=\frac{n !}{\alpha ! \beta ! \gamma !} c_{x}^{\alpha} c_{y}^{\beta} c_{z}^{\gamma}
$$

We are now in a position to tackle the problem of velocity, first when the Hamiltonian $\widehat{H}$ takes into account the SOI, and, second, when the Hamiltonian $\widehat{H}_{\text {eff }}$ is an effective Hamiltonian.

\section{B. Velocity operator in presence of SOI interaction}

It is usually admitted that the velocity operator $\widehat{\mathbf{v}}$ is equal to $\partial \widehat{\mathcal{H}} / \partial \widehat{\mathbf{p}}$ whatever the Hamiltonian $\widehat{\mathcal{H}}$. However, to the best of our knowledge, the derivation can be found only when $\widehat{\mathcal{H}}$ is quadratic in $\widehat{\mathbf{p}}$. Therefore a general derivation, in particular in the case of effective Hamiltonians, is mandatory. We start from Ehrenfest's theorem (valid whatever the Hamiltonian $\widehat{\mathcal{H}}$ )

$$
\langle\widehat{\mathbf{v}}\rangle=\frac{\mathrm{d}\langle\widehat{\mathbf{r}}\rangle}{\mathrm{d} t}=\frac{i}{\hbar}\langle[\widehat{\mathcal{H}}, \widehat{\mathbf{r}}]\rangle
$$

If $(i / \hbar)\langle[\widehat{\mathcal{H}}, \widehat{\mathbf{r}}]\rangle=\langle\partial \widehat{\mathcal{H}} / \partial \mathbf{p}\rangle$, then $\widehat{\mathbf{v}}=\partial \widehat{\mathcal{H}} / \partial \widehat{\mathbf{p}}$ because two linear operators which have the same mean values over all possible states are equal: $\langle\widehat{A}\rangle=\langle\widehat{B}\rangle$ implies that $\widehat{A}=\widehat{B} .^{20}$ Practically, it is enough to show that $(i / \hbar)[\widehat{\mathcal{H}}, \widehat{\mathbf{r}}]=\partial \widehat{\mathcal{H}} / \partial \widehat{\mathbf{p}}$ to prove that $\widehat{\mathbf{v}}=\partial \widehat{\mathcal{H}} / \partial \widehat{\mathbf{p}}$.

First, considering the case $\widehat{\mathcal{H}}=\widehat{H}_{0}$ which contains a $\widehat{H}^{(2)}$ term (Eq. 1), $(i / \hbar)\left[\widehat{H}_{0}, \widehat{\mathbf{r}}\right]=$ $(\hbar / i m) \widehat{\mathbf{p}}=\partial \widehat{H}_{0} / \partial \widehat{\mathbf{p}}$, for a system described by an Hamiltonian quadratic versus momentum components, and we obtain the velocity $\widehat{\mathbf{v}}_{0}$ :

$$
\widehat{\mathbf{v}}_{0}=\frac{\partial \widehat{H}_{0}}{\partial \widehat{\mathbf{p}}} .
$$

Second, we have to check that this relation still holds in the presence of SOI where the Hamiltonian is $\widehat{\mathcal{H}}=\widehat{H}=\widehat{H}_{0}+\widehat{H}_{S O}$ (Eq. 3). In other words we want to show that

$$
\widehat{\mathbf{v}}=\frac{\partial \widehat{H}}{\partial \widehat{\mathbf{p}}} .
$$

We know that $\widehat{\mathbf{v}}_{0}=(i / \hbar)\left[\widehat{H}_{0}, \widehat{\mathbf{r}}\right]=\partial \widehat{H}_{0} / \partial \widehat{\mathbf{p}}$. To show that Eq. 34 is valid, it is enough to show that $\widehat{\mathbf{v}}_{S O}=\partial \widehat{H}_{S O} / \partial \widehat{\mathbf{p}}$, which will give $\widehat{\mathbf{v}}=\partial \widehat{H} / \partial \widehat{\mathbf{p}}$ with $\widehat{\mathbf{v}}=\widehat{\mathbf{v}}_{0}+\widehat{\mathbf{v}}_{S O}$. A straightforward 
calculation yields

$$
\widehat{\mathbf{v}}_{S O}=\frac{i}{\hbar}\left[\widehat{H}_{S O}, \widehat{\mathbf{r}}\right]=\frac{\hbar}{4 m^{2} c^{2}}(\widehat{\sigma} \times \nabla \mathfrak{U})=\frac{\partial \widehat{H}_{S O}}{\partial \widehat{\mathbf{p}}}
$$

which proves Eq. 34: the derivative of the Hamiltonian, with respect to the momentum operator, still provides a suitable definition of the velocity when the SOI term is taken into account.

\section{Velocity operator with an effective Hamiltonian $\widehat{H}_{e f f}$}

We generalize the results obtained in Sec. IIIB, to the case of a generic effective Hamil-

tonian $\widehat{\mathcal{H}}=\widehat{H}_{\text {eff }}$. Again we exploit Ehrenfest's theorem, as written in Eq. 32. Considering for instance the $x$ component, we verify that

$$
\left[\widehat{H}^{(n)}, x\right]=\sum_{\alpha+\beta+\gamma=n} c^{\alpha \beta \gamma}\left(\frac{\hbar}{i}\right) \alpha \widehat{p}_{x}^{\alpha-1} \widehat{p}_{y}^{\beta} \widehat{p}_{z}^{\gamma}=\frac{\hbar}{i} \frac{\partial \widehat{H}^{(n)}}{\partial \widehat{p}_{x}}
$$

or

$$
\frac{i}{\hbar}\left[\widehat{H}_{\mathbf{p}}, \widehat{\mathbf{r}}\right]=\frac{i}{\hbar}\left[\widehat{H}_{\mathrm{eff}}, \widehat{\mathbf{r}}\right]=\frac{\partial \widehat{H}_{e f f}}{\partial \widehat{\mathbf{p}}}
$$

which proves that

$$
\widehat{\mathbf{v}}=\frac{\partial \widehat{H}_{e f f}}{\partial \widehat{\mathbf{p}}}
$$

Using Eqs. 28, 31, it is then easy to calculate the $j$ component $\widehat{v}_{j}^{(n)}(j=x, y, z)$ of the velocity operator $\widehat{\mathbf{v}}^{(n)}$ associated to $\widehat{H}^{(n)}$ :

$$
\widehat{v}_{j}^{(n)}=\frac{\partial \widehat{H}^{(n)}}{\partial \widehat{p}_{j}}=n c_{j}\left(c_{x} \widehat{p}_{x}+c_{y} \widehat{p}_{y}+c_{z} \widehat{p}_{z}\right)^{n-1} .
$$

We introduce the scalar product between the momentum $\widehat{\mathbf{p}}$ and the velocity operator $\widehat{\mathbf{v}}^{(n)}$

$$
\widehat{p}_{x} \widehat{v}_{x}^{(n)}+\widehat{p}_{y} \widehat{v}_{y}^{(n)}+\widehat{p}_{z} \widehat{v}_{z}^{(n)}=n\left(c_{x} \widehat{p}_{x}+c_{y} \widehat{p}_{y}+c_{z} \widehat{p}_{z}\right)^{n}=n \widehat{H}^{(n)}
$$

With this notation, $\widehat{\mathbf{v}}_{0}$, introduced in the paragraph IIIB, is such that $\widehat{\mathbf{v}}_{0}=\widehat{\mathbf{v}}^{(2)}$. Eq. 40 means that

$$
\widehat{\mathbf{p}} \cdot \widehat{\mathbf{v}}^{(n)}=n \widehat{H}^{(n)}
$$

and eventually

$$
\widehat{H}_{e f f} \psi=\left(\widehat{\mathbf{p}} \cdot \sum_{n} \frac{1}{n} \widehat{\mathbf{v}}^{(n)}\right) \psi+V \psi=E \psi
$$


As pointed out in Sec. III, we are allowed to define current operators in open systems provided that we properly take into account their boundary conditions. In Appendix B, we show that performing the proper symmetrization according to the following rule (Eq. 44), we find a probability current operator that for the $j^{\text {th }}$-Cartesian component reads:

$$
\begin{aligned}
& \widehat{J}_{j}\left(\mathbf{r}_{0}\right)=\sum_{n} \widehat{J}_{j}^{(n)}\left(\mathbf{r}_{0}\right) \\
& \widehat{J}_{j}^{(n)}\left(\mathbf{r}_{\mathbf{0}}\right)=\sum_{\substack{l(k) \in\{x, y, z\} \\
k=1, \ldots, n-1}} c_{j, l(1), \cdots, l(n-1)}\left[\delta_{\mathbf{r}_{0}} \widehat{p}_{l(1)} \ldots \widehat{p}_{l(n-1)}+\widehat{p}_{l(1)} \delta_{\mathbf{r}_{0}} \ldots \widehat{p}_{l(n-1)}+\ldots .+\widehat{p}_{l(1)} \ldots \widehat{p}_{l(n-1)} \delta_{\mathbf{r}_{0}}\right]
\end{aligned}
$$

where $\delta_{\mathbf{r}_{0}}=\delta\left(\mathbf{r}-\mathbf{r}_{0}\right)$ is the Dirac distribution. We must still verify that the divergence of the current, calculated with the operator defined by Eq. 44, satisfies the conservation equation for the density of probability (Eq. 14 when $\widehat{A}$ is the identity). It is straightforward to show (see Appendix B) that the divergence of the probability current can be written as:

$$
\nabla \cdot \mathbf{J}[\psi]=\sum_{n} \nabla \cdot \mathbf{J}^{(n)}[\psi]=-\frac{2}{\hbar} \operatorname{Im} \sum_{n} \sum_{j=\{x, y, z\}} \sum_{\substack{l(k) \in\{x, y, z\} \\ k=1, ., n-1}} c_{j, l(1), \cdots, l(n-1)}\left(\psi\left|\widehat{p}_{j} \widehat{p}_{l(1)} \ldots \widehat{p}_{l(n-1)}\right| \psi\right)
$$

where we again use the notations $(\psi \mid \widehat{A} \psi)=\psi^{\dagger} \widehat{A} \psi$ as in Ref. 2. Then, we recover all the terms of Eq. 14, so that Eq. 44 indeed provides a correct definition of the current operator. Obviously, adding a term proportional to the curl of any vector field would not affect the result. Such a definition of $\hat{\mathbf{J}}$ provides an unambiguous and general tool for evaluating the probability current. Provided the Hamiltonian of the whole system is known, this probability-current operator guarantees the requirements of the continuity equation.

Now it is useful to introduce the Hermitian symmetrized velocity operator

$$
\widehat{\mathfrak{v}}_{j}^{(n)}\left(\mathbf{r}_{\mathbf{0}}\right)=\frac{n}{2} \sum_{\substack{l(k) \in\{x, y, z\} \\ k=1, ., n-1}} c_{j, l(1), \cdots, l(n-1)}\left[\delta_{\mathbf{r}_{0}} \widehat{p}_{l(1) \cdots \widehat{p}_{l(n-1)}}+\widehat{p}_{l(1) \cdots \widehat{p}_{l(n-1)}} \delta_{\mathbf{r}_{0}}\right]
$$

For example for $n \geq 2$, the comparison between Eqs. 44 and 46 clearly shows that $\widehat{J}_{j}^{(n)}\left(\mathbf{r}_{\mathbf{0}}\right)$ contains $n-2$ extra terms, which are straightforwardly obtained from $\partial \widehat{H}_{e f f} / \partial \widehat{\mathbf{p}}$. For instance, with $\widehat{H}_{e f f} \equiv \widehat{p}^{n}$, we have $\partial \widehat{H}_{e f f} / \partial \widehat{\mathbf{p}} \equiv n \widehat{p}^{n-1}$, so that $\widehat{\mathfrak{v}}^{(n)}\left(\mathbf{r}_{\mathbf{0}}\right) \equiv$ $(n / 2)\left(\delta_{\mathbf{r}_{0}} \widehat{p}^{n-1}+\widehat{p}^{n-1} \delta_{\mathbf{r}_{0}}\right)$, whereas $\widehat{J}^{(n)}\left(\mathbf{r}_{\mathbf{0}}\right) \equiv\left(\delta_{\mathbf{r}_{0}} \widehat{p}^{n-1}+\widehat{p} \delta_{\mathbf{r}_{0}} \widehat{p}^{n-2}+\ldots+\widehat{p}^{n-1} \delta_{\mathbf{r}_{0}}\right)$. As shown in Ref. 2, extra terms are specially important for evanescent waves. Therefore, in the following we deal with tunneling problems. 


\section{BenDaniel-Duke-LIKE FORMULATION AND BOUNDARY CONDITIONS}

We stress that the central question when defining the current operators and related quantities is the proper definition of the system and of its boundaries. Dealing with heterojunctions, where each bulk medium is described by the relevant Hamiltonian, requires defining proper matching conditions at the boundaries. In this sense, the BDD Hamiltonian 17 is the simplest smart approach that allows solution of the Schrödinger equation over the whole space while it guaranties the conservation of the probability current at the interface. The principle is the following. Let us consider a one-dimensional problem and two different media for $x<0$ and $x>0$. Each medium is characterized by its own Hamiltonian. The question is to find a solution of the Schrödinger equation, made of eigenfunctions of the relevant band of the two bulk materials, which ensures the continuity of the probability current at the origin. In this sense, the problem is analogous to a scattering problem, where the wave functions are determined only at some distance of the scattering potential. Proper matching conditions relevant to the extension of the bulk envelope functions at the origin will allow

one to determine the envelope function over the whole space. For that, BBD propose writing an Hamiltonian over the whole space as $\widehat{p}_{x}[1 / 2 m(x)] \widehat{p}_{x}+V(x)$ where $m(x)$ is the effective mass in each medium. The integration of this BDD Hamiltonian around the boundary automatically ensures the continuity of the probability current of Eq. 2, provided that $\psi(x)$ and $[1 / m(x)][\partial \psi / \partial x]$ are continuous.

Now, consider two regions (1) and (2) and assume that each region is made of a given crystalline material. We look for the envelope function, solution of the Schrödinger equation, which is made from plane waves which are eigenstates of the crystal, inside each material. Observe that, near the interface, the crystal periodicity is broken so that the true Hamiltonian and the true eigenfunctions will become involved. The principle is then to define proper matching conditions applying to the prolongation of the envelope function at the origin. For that purpose, we consider a volume $\mathbb{V}$, limited by a surface $\mathbb{S}$, that surrounds an interface portion. Similarly to the BDD technique, we start from Eq. 42 and we integrate the Schrödinger equation over $\mathbb{V}$. Using Ostrogradski's theorem, when $\mathbb{V}$ tends to zero, we obtain

$$
\lim _{\mathbb{V} \rightarrow 0} \int_{\mathbb{S}}\left(\sum_{n} \frac{1}{n} \mathbf{v}^{(n)} \psi\right) \cdot \mathrm{d} \mathbf{s}=0
$$

where ds is normal to the surface $\mathbb{S}$. 
For a one dimensional case with the interface at the origin, Eq. 47 becomes:

$$
\lim _{\varepsilon \rightarrow 0}\left[\sum_{n} \frac{1}{n} v^{(n)} \psi\right]_{-\varepsilon}^{+\varepsilon}=0
$$

Let us again emphasize that no information is obtained on the true wave function near the origin. Eq. 48 does not ensure either the continuity of the envelope function or the existence of derivatives at the interface.

As an illustration, let us consider the case of a Rashba Hamiltonian

$$
\hat{H}_{e f f}=a \widehat{p}+b \widehat{p}^{2}
$$

where $a$ and $b$ are two Hermitian matrices. According to Eq. 48, we can write down the first continuity condition as follows:

$$
[a \psi+b \widehat{p} \psi]_{-\varepsilon}^{+\varepsilon}=0
$$

Using this condition to solve the problem, and adding a priori the continuity of the envelope function at the interface as a second condition, we verify that the probability current is indeed continuous at the interface:

$$
J[\psi]=\langle\psi|a+b \widehat{p}| \psi\rangle+c . c .
$$

Then, the jump of the derivative of the wave function at the interface is determined by

$$
[b \widehat{p} \psi]_{-\varepsilon}^{+\varepsilon}=-[a]_{-\varepsilon}^{+\varepsilon} \psi(0)
$$

It is clear then that the BDD approach, introduced to solve a problem with a quadratic Hamiltonian, is also suitable to obtain a solution when a Rashba contribution is added; then we can say that up to the second order in the momentum-power series expansion of the Hamiltonian, the continuity of a "generalized velocity" (see Eq. 48) and the continuity of the wave function at the interface imply the conservation of the probability current at this point. Remarkably, the boundary conditions that we need to solve the problem drastically change when moving to the case of a DP Hamiltonian with cubic terms. The crucial point, that we address in the following, is that we cannot make any hypothesis about the continuity of the wave function because, if we need to ensure probability-current conservation at an interface, we must accept an envelope function $\psi$ which is no longer continuous. 
To give an insight into the expression of the current operator and into the conservation of the probability current, let us again come back to an interface between two semi-infinite one-dimensional media (1) and (2). In each bulk crystal, the relevant Hamiltonian is

$$
\widehat{H}_{r}=\sum_{n} \widehat{H}_{r}^{(n)}+V_{r}
$$

with

$$
\widehat{H}_{r}^{(n)}=\gamma_{r}^{(n)} \widehat{p}^{n}
$$

with $r=1$ or $r=2$ depending on wether $x<0$ or $x>0$. $\widehat{H}_{r}$ admits the eigenfunctions $\varphi_{r}$, associated to the fixed energy $E$ which verify

$$
\widehat{H}_{r} \varphi_{r}=E \varphi_{r}
$$

Near the heterojunction, the spatial periodicity is broken, so that over a few Wigner-Seitz cells, the electron states are no longer pure Bloch states. We consider two coordinates, $-w_{1}$ and $w_{2}$, so that, in the regions $\left.]-\infty,-w_{1}\right]$ and $\left[w_{2},+\infty[\right.$ the electronic structure remains unaffected. In these regions, the Hamiltonian writes

$$
\widehat{\mathfrak{H}}=\Theta\left(-x-w_{1}\right) \widehat{H}_{1}+\Theta\left(x-w_{2}\right) \widehat{H}_{2}
$$

where $\Theta(x)$ is the Heaviside function. In the vicinity of the heterojunction, ]- $-w_{1}, w_{2}[$, the form of the Hamiltonian and of the wave functions are not explicitly known. We consider a wave function $\psi$ which is an eigenstate of the Hamiltonian over the whole space at energy $E$. We expect that, over the domain $\left.]-\infty,-w_{1}\right] \cup\left[w_{2},+\infty[, \psi\right.$ coincides with $\Psi=\Theta\left(-x-w_{1}\right) \varphi_{1}+\Theta\left(x+w_{2}\right) \varphi_{2}$. We thus expect the following relation to be satisfied

$$
\left\langle\varphi_{1} \Theta(-x)+\varphi_{2} \Theta(x)|\widehat{\mathfrak{H}}| \Psi\right\rangle=E\left[\left\langle\varphi_{1} \mid \varphi_{1}\right\rangle_{(1)}+\left\langle\varphi_{2} \mid \varphi_{2}\right\rangle_{(2)}\right]
$$

where $\langle\mid\rangle_{(r)}$ means summation over the bulk part of region $(r)$. Observe that:

$$
\begin{aligned}
\widehat{H}_{1}^{(n)} \Psi & =\gamma_{1}^{(n)} \widehat{p}^{n} \Theta\left(-x-w_{1}\right) \varphi_{1}+V_{1} \Theta\left(-x-w_{1}\right) \varphi_{1} \\
& =\Theta\left(-x-w_{1}\right)\left(\gamma_{1}^{(n)} \widehat{p}^{n}+V_{1}\right) \varphi_{1} \\
& +i \hbar \gamma_{1}^{(n)}\left[\delta\left(x+w_{1}\right) \widehat{p}^{n-1}+\widehat{p} \delta\left(x+w_{1}\right) \widehat{p}^{n-2}+\ldots+\widehat{p}^{n-1} \delta\left(x+w_{1}\right)\right] \varphi_{1} \\
& =\Theta\left(-x-w_{1}\right)\left(\gamma_{1}^{(n)} \widehat{p}^{n}+V_{1}\right) \varphi_{1}+i \hbar \widehat{J}_{1}^{(n)} \varphi_{1}=\Theta\left(-x-w_{1}\right) E \varphi_{1}+i \hbar \widehat{J}_{1}^{(n)} \varphi_{1}
\end{aligned}
$$


and similarly

$$
\widehat{H}_{2}^{(n)} \Psi=\Theta\left(x+w_{2}\right) E \varphi_{2}-i \hbar \widehat{J}_{2}^{(n)} \varphi_{2}
$$

So that

$$
\begin{aligned}
\left\langle\varphi_{1} \Theta(-x)+\varphi_{2} \Theta(x)|\widehat{\mathfrak{H}}| \Psi\right\rangle & =E\left[\left\langle\varphi_{1} \mid \varphi_{1}\right\rangle_{(1)}+\left\langle\varphi_{2} \mid \varphi_{2}\right\rangle_{(2)}\right] \\
& +i \hbar\left(\widehat{J}_{2}^{(n)}\left[\varphi_{2}\left(w_{2}\right)\right]-\widehat{J}_{1}^{(n)}\left[\varphi_{1}\left(-w_{1}\right)\right]\right) \\
& =E\left[\left\langle\varphi_{1} \mid \varphi_{1}\right\rangle_{(1)}+\left\langle\varphi_{2} \mid \varphi_{2}\right\rangle_{(2)}\right] .
\end{aligned}
$$

This implies that

$$
\widehat{J}_{2}^{(n)}\left[\varphi_{2}\left(w_{2}\right)\right]=\widehat{J}_{1}^{(n)}\left[\varphi_{1}\left(-w_{1}\right)\right]
$$

The important point is not the conservation relation, which might appear as physically obvious, but that, in Eqs. 58 and 59, the symmetrized current operator is automatically generated in the form derived in Eq. 44, providing a physical insight into this mathematical expression. Because we only deal with wave functions at some distance from the heterojunction, the continuity of the true wave function at $x=0$ does not implies the continuity of the envelope function $\Psi$ which may be discontinuous. This is in line with the considerations of Harrison. $\stackrel{18}{ }$ Eq. 48 and 61 generate a set of boundary conditions relevant to the tunneling problem.

\section{THE [110]-ORIENTED GaAs BARRIER}

We analyze the case of electron tunneling under normal incidence through a GaAs [110]oriented barrier, which was shown to be non trivial and solved in special cases in Ref. 2 . Hereafter, we apply the tools and boundary conditions presented in this paper to solve it in a more simple and general manner. We confirm and generalize the results derived in Ref. 2. In particular, we are able to solve the problem of an heterojunction between a free-electron media and a semiconductor without inversion center, where the DP field is a step function, which remained puzzling. In the [110] direction, the DP Hamiltonian is:

$$
\widehat{H}_{D P}=\frac{\gamma_{c}}{\hbar^{2}} \widehat{p}^{2} \pm \frac{\gamma}{2 \hbar^{3}} \widehat{p}^{3}
$$

where $+(-)$ refers to the up (down)- spin channel quantized along the DP field direction. We consider as solution a general wavefunction written as follows:

$$
\psi=\alpha\left(\psi_{0}+\frac{i \beta}{\hbar \gamma_{c} k^{2}} \gamma_{c} \widehat{p} \psi_{0}\right) e^{i \chi z}
$$


where $\psi_{0}$ is the zeroth order function that is a solution of the tunneling problem with energy $E$ and with the potential $V$ when SOI is turned off. Here $\gamma_{c} k^{2}=E-V, \alpha$ and $\beta$ are complex parameters to be determined, and $\chi$ is a real (see below) wavevector component which is added to $k$ when SOI is turned on. We have the relations

$$
\begin{gathered}
\widehat{p} \psi=\alpha\left(\widehat{p} \psi_{0}+i \frac{\beta}{\hbar \gamma_{c} k^{2}} \gamma_{c} \widehat{p}^{2} \psi_{0}\right) e^{i \chi z}+\hbar \chi \psi, \\
\widehat{p}^{2} \psi=\hbar^{2}\left(\frac{E-V}{\gamma_{c}}-\chi^{2}\right) \psi+2 \hbar \chi \widehat{p} \psi .
\end{gathered}
$$

We calculate the velocity operators from Eq. 39

$$
\frac{1}{2} v^{(2)}+\frac{1}{3} v^{(3)}=\frac{\gamma_{c}}{\hbar^{2}} \widehat{p} \pm \frac{\gamma}{2 \hbar^{3}} \widehat{p}^{2}
$$

and, according to Eq. 48, we find the matching condition

$$
\left[\gamma_{c}\left(1 \pm \frac{\gamma}{\gamma_{c}} \chi\right) \widehat{p} \psi\right]_{-\varepsilon}^{+\varepsilon}=\mp\left[\frac{1}{2} \gamma \hbar\left(\frac{E-V}{\gamma_{c}}-\chi^{2}\right) \psi\right]_{-\varepsilon}^{+\varepsilon}
$$

which is a generalization of Eq. (3.50) of Ref. 2.

Now, we have to satisfy the conservation of the probability current

$$
J[\psi]=\frac{\gamma_{c}}{\hbar^{2}}\left(1 \pm \frac{\gamma}{\gamma_{c}} \chi\right)\left[\psi^{*} \widehat{p} \psi+\psi(\widehat{p} \psi)^{*}\right] \pm \frac{\gamma}{\hbar}\left(\frac{E-V}{\gamma_{c}}-\chi^{2}\right)|\psi|^{2} \pm \frac{\gamma}{2 \hbar^{3}}|\widehat{p} \psi|^{2} .
$$

We obtain here an important result: The envelope function cannot be continuous at the interface. Indeed, assume $\psi$ to be continuous. Then, after Eq. 67, we see that the last term in Eq. 68, that we rewrite as $\pm\left(1 / 2 \hbar^{3}\right)\left(\gamma / \gamma_{c}^{2}\right) \gamma_{c}^{2}|\widehat{p} \psi|^{2}$ must be continuous. This is not possible since $\gamma_{c} \widehat{p} \psi_{0}$ is continuous (unless $\gamma / \gamma_{c}^{2}$ is almost continuous, which would be fortuitous).

We have to determine $\psi$ complying the boundary conditions, which is not simple because the expression providing the current is not a linear function of $\psi$. However, if we consider $\gamma$ as a first-order quantity and look for a solution to first order only, the result is surprisingly simple, as shown below. From the Schrödinger equation - Eq. 62 -, we find that $\chi$ verifies

$$
\gamma_{c}\left(2 k \chi+\chi^{2}\right) \pm \frac{\gamma}{2}\left(k^{3}+3 k^{2} \chi+3 k \chi^{2}+\chi^{3}\right)=0
$$

then

$$
\chi \simeq \mp \frac{1}{4} \frac{\gamma}{\gamma_{c}} k^{2}=\mp \frac{1}{4} \frac{\gamma}{\gamma_{c}} \frac{E-V}{\gamma_{c}}
$$


As stated above, $\chi$ is a real quantity. For each spin, there are two others roots of the cubic equation (Eq. 69) which are much larger than the width of the Brillouin zone ; These two roots are of the order of $\gamma_{c} / \gamma$ which is about $2 \AA$ (two times the Brillouin zone width) in GaAs (see Fig. 4 of Ref. 2) and have no physical meaning. Note that, the cubic DP term, obtained from perturbation expansion, only holds for small wave vectors, a few percent of the Brillouin zone, so that taking into account these two other roots would be meaningless. From Eq. 63, we see that, upon tunneling, the up- and down- spin electrons undergo opposite phase shifts, which is equivalent to a precession around the DP-field direction. This would be quite intuitive if the field were not a complex quantity, and constitutes a prediction which can be experimentally tested. Let us calculate the current at the interface $J[\psi(0)]$ to first order

$$
\begin{aligned}
J[\psi(0)]=|\alpha|^{2} \frac{\gamma_{c}}{\hbar^{2}}\left[\psi_{0}^{*} \widehat{p} \psi_{0}+\psi_{0}\left(\widehat{p} \psi_{0}\right)^{*}\right]+ & \frac{\gamma_{c}}{2 \hbar}\left[2|\alpha|^{2}(\chi-\operatorname{Im} \beta) \pm \frac{\gamma}{\gamma_{c}} \frac{E-V}{\gamma_{c}}\right]\left|\psi_{0}\right|^{2} \\
& \left.+\frac{\gamma_{c}}{\hbar^{3}}\left[-|\alpha|^{2} \frac{2 \gamma_{c}}{E-V} \operatorname{Im} \beta \mp \frac{\gamma}{2 \gamma_{c}}\right]\left|\widehat{p} \psi_{0}\right|^{2}\right\}
\end{aligned}
$$

where the values of $\psi_{0}$ and of its derivative are calculated at the origin. Observe that with the choice

$$
|\alpha|^{2}=1 \quad \text { and } \quad \operatorname{Im} \beta=-\chi
$$

the second and the last terms of Eq. 71 vanish so that

$$
J[\psi(0)]=\frac{\gamma_{c}}{\hbar^{2}}\left[\psi_{0}^{*} \widehat{p} \psi_{0}+\psi_{0}\left(\widehat{p} \psi_{0}\right)^{*}\right]=J^{f}[\psi(0)]
$$

where $J^{f}[\psi(0)]$ results from the application of the free-electron current operator on $\psi(0)$. Thus, we obtain another essential result: To first order, turning on the SOI does not alter the value of the probability current. Consequently, to solve the problem we have only to show that $\psi$, given by Eq. 63 and with the conditions defined in Eq. 72, can match the boundary condition expressed by Eq. 67. We obtain

$$
\gamma_{c}[\alpha \widehat{p}+\hbar(i \alpha \beta-\chi)] \psi_{0}=\gamma_{c}[\alpha \widehat{p}+\hbar[\alpha(i \operatorname{Re} \beta+\chi)-\chi]] \psi_{0} \quad \text { continuous. }
$$

The continuity of Eq. 74 can always be ensured by taking $\alpha=1$ and $\operatorname{Re} \beta=0$. Then, we calculate $\psi$ according to Eq. 63 and deduce the jump of $\psi$ at the interface

$$
[\psi(0)]_{0-}^{0+}=\left[\frac{\chi}{\hbar(E-V)}\right]_{0-}^{0+}\left(\gamma_{c} \widehat{p} \psi_{0}\right)=\mp \frac{1}{4 \hbar}\left[\frac{\gamma}{\gamma_{c}^{2}}\right]_{0-}^{0+}\left(\gamma_{c} \widehat{p} \psi_{0}\right) .
$$




\section{SPIN CURRENT}

We have shown in Sec. V that Eq. 44 provides a general and symmetrized definition of the probability-current operator. Following the conceptual scheme developed in Ref. 12, we can define the spin currents in the up- and down-spin channels by taking $\widehat{A}=\widehat{\pi}_{s}$, where $\widehat{\pi}_{s}$ is the orthogonal projector on the spin basis $(s= \pm)$. Then the $\mathrm{SC}$ current $\delta \mathbf{J}_{\mathbf{u}, j}[\psi]$, that arises from the difference between the up-spin and the down-spin current, is obtained by taking $\widehat{A}=\widehat{\sigma}_{\mathbf{u}}$, the Pauli operator along the $\mathbf{u}$ direction defining the quantization axis. It is straightforward to see that, as in Ref. 12, the $j$-component of the spin-current operator is obtained from the $j$-component of the probability-current operator after the substitution

$$
c_{j, l(1), \ldots, l(n)}^{\prime}=\frac{1}{2}\left\{\sigma_{\mathbf{u}}, c_{j, l(1), \ldots, l(n)}\right\}
$$

\section{CONCLUSION}

We have proposed a systematic procedure to construct properly-symmetrized current operators. We have extended the BenDaniel Duke approach $\frac{17}{}$ to deal with heterostructures where SOI is included, introducing generalized boundary conditions, which allow us to consider open systems. We have shown that up to second order, usual matching conditions and in particular the continuity of the envelope function at an interface, yield solutions which comply with the conservation of the probability current. This no longer holds as soon as a cubic term is included (D'yakonov-Perel' term). We have illustrated our findings on the model case of a [110]-oriented GaAs barrier, which had already been addressed in Ref. 2. We recover and generalize the results of Ref. 2 in a more simple and direct treatment. The tools we have developed can be applied to the holes in the valence bands or to the electrons in the conduction band so that they should be important for semiconductor-based spintronics.

\section{Acknowledgments}

We are deeply indebted to Travis Wade for a careful reading of the manuscript. 


\section{Appendix A: Symmetry properties of current operators}

In Sec. Q Eq. 10, we derived the local form of the Ehrenfest theorem for a general operator $\widehat{A}$ and deduced the expression of the associated current $\mathbf{J}_{A}$. First, consider the case where $\widehat{A}=\widehat{I}$, where $\widehat{I}$ is the identity and the quadratic Hamiltonian $\widehat{\mathbf{p}}^{2} / 2 m$. We rewrite Eq. 10 as

$$
\frac{\partial}{\partial t}|\psi|^{2}=-\nabla \cdot \operatorname{Re}\left(\psi^{\dagger} \frac{\widehat{\mathbf{p}}}{m} \psi\right)=-\nabla \cdot \mathbf{J}[\psi]
$$

We recover the usual expression for the free-electron probability current

$$
\mathbf{J}[\psi]=\operatorname{Re}\left(\psi^{\dagger} \frac{\widehat{\mathbf{p}}}{m} \psi\right) .
$$

Note that:

$$
\frac{\partial}{\partial t}|\psi|^{2}=\frac{1}{i \hbar}\left[\left(\psi^{\dagger} \frac{\widehat{\mathbf{p}}^{2}}{2 m} \psi\right)-\left(\psi^{\dagger} \frac{\widehat{\mathbf{p}}^{2}}{2 m} \psi\right)^{*}\right]=\frac{1}{i \hbar}\left[\left(\psi^{\dagger} \frac{\widehat{\mathbf{p}}^{2}}{2 m} \psi\right)-\left(\widehat{K}_{0} \psi\right)^{\dagger} \frac{\widehat{\mathbf{p}}^{2}}{2 m}\left(\widehat{K}_{0} \psi\right)\right]
$$

where $\widehat{K}_{0}$ is the time-reversal Kramers operator for a spinless particle, which consists of taking the complex conjugate in the $\mathbf{r}$-representation. Let us check the expression of the current operators we defined under time inversion symmetry. For this purpose we consider the term

$-2 i \hbar \nabla \cdot \mathbf{J}_{A}=2 i \operatorname{Im}\left(\psi^{\dagger}\{\widehat{A}, \widehat{H}\} \psi\right)=\left[\psi^{\dagger} \widehat{A} \widehat{H} \psi-\left(\psi^{\dagger} \widehat{A} \widehat{H} \psi\right)^{*}\right]+\left[\psi^{\dagger} \widehat{H} \widehat{A} \psi-\left(\psi^{\dagger} \widehat{H} \widehat{A} \psi\right)^{*}\right]$.

First, look at the term $\psi^{\dagger} \widehat{A} \widehat{H} \psi$

$$
\begin{aligned}
(\widehat{K} \psi \mid \widehat{A} \widehat{H} \widehat{K} \psi) & =\left(\widehat{K}_{0} \psi \mid \widehat{R}^{\dagger} \widehat{A} \widehat{H} \widehat{K} \psi\right)=\left(\widehat{K}_{0} \psi \mid \widehat{R}^{\dagger} \widehat{A} \widehat{K} \widehat{H} \psi\right)=-\varepsilon_{A}\left(\widehat{K}_{0} \psi \mid \widehat{R}^{\dagger} \widehat{K} \widehat{A} \widehat{H} \psi\right) \\
& =-\varepsilon_{A}\left(\widehat{K}_{0} \psi \mid \widehat{K}_{0} \widehat{A} \widehat{H} \psi\right)=-\varepsilon_{A}(\psi \mid \widehat{A} \hat{H} \psi)^{*}
\end{aligned}
$$

Here, $\widehat{K}=\widehat{R} \widehat{K}_{0}$ is the Kramers operator for a particle with spin $1 / 2, \widehat{R}=-i \sigma_{y}$ $\left(\widehat{R}^{\dagger}=\widehat{R}^{-1}\right)$, and $\varepsilon_{A}= \pm 1$ depending wether $\widehat{A}$ verifies $\underline{21}$

$$
\widehat{K} \widehat{A} \widehat{K}=\varepsilon_{A} \widehat{A} \quad \text { or } \quad \widehat{R}^{\dagger} \widehat{A} \widehat{R}=\varepsilon_{A} \widehat{A}^{*} .
$$

Similarly, for the term $\psi^{\dagger} \widehat{H} \widehat{A} \psi$

$$
\begin{aligned}
(\widehat{K} \psi \mid \widehat{H} \widehat{A} \widehat{K} \psi) & =-\varepsilon_{A}\left(\widehat{K}_{0} \psi \mid \widehat{R}^{\dagger} \widehat{K} \widehat{H} \widehat{A} \psi\right) \\
& =-\varepsilon_{A}\left(\widehat{K}_{0} \psi \mid \widehat{K}_{0} \widehat{H} \widehat{A} \psi\right)=-\varepsilon_{A}(\psi \mid \widehat{H} \widehat{A} \psi)^{*}
\end{aligned}
$$


Thus, we obtain

$$
2 i \operatorname{Im}\left(\psi^{\dagger}\{\widehat{A}, \widehat{H}\} \psi\right)=\psi^{\dagger}\{\widehat{A}, \widehat{H}\} \psi+\varepsilon_{A}(\widehat{K} \psi)^{\dagger}\{\widehat{A}, \widehat{H}\}(\widehat{K} \psi)
$$

We conclude that the general expression for the current of $\widehat{A}$ is

$$
\nabla \cdot \mathbf{J}_{A}=-\frac{1}{2 i \hbar}\left[\psi^{\dagger}\{\widehat{A}, \widehat{H}\} \psi+\varepsilon_{A}(\widehat{K} \psi)^{\dagger}\{\widehat{A}, \widehat{H}\}(\widehat{K} \psi)\right]
$$

\section{Appendix B: Complete derivation of the current operator $\hat{\mathbf{J}}$}

We are interested in finding the form of the current operator $\widehat{\mathbf{J}}=\left(\widehat{J}_{x}, \widehat{J}_{y}, \widehat{J}_{z}\right)$ for an Hamiltonian $\widehat{H}^{(n)}+V(\mathbf{r})$ - the current operator being $\widehat{\mathbf{J}}^{(n)}$ - and more generally for the Hamiltonian $\widehat{H}_{e f f}=\widehat{H}_{\mathbf{p}}+V(\mathbf{r})=\sum_{n} \widehat{H}^{(n)}+V(\mathbf{r})$ (Eqs. 26-27) - the current operator being $\widehat{\mathbf{J}}$. For an Hamiltonian $\widehat{\mathbf{p}}^{2} / 2 m+V(\mathbf{r})$, it is known 22 that the $j^{\text {th }}$ component of the current operator $(j=x, y$, or $z)$ at the point $\mathbf{r}_{0}$ is of the shape $\widehat{J}_{j}^{(2)}\left(\mathbf{r}_{0}\right)=$ $(1 / 2 m)\left[\delta_{\mathbf{r}_{0}} \widehat{p}_{j}+\widehat{p}_{j} \delta_{\mathbf{r}_{0}}\right]$; With the notation of Eqs. 26ㄴㄹ, $\widehat{H}^{(2)}=\sum_{\substack{l(k) \in\{x, y, z\} \\ k=1,2}} c_{l(1), l(2)} \widehat{p}_{l(1)} \widehat{p}_{l(2)}$, $\widehat{J}_{j}^{(2)}\left(\mathbf{r}_{0}\right)=\sum_{l(1)=\{x, y, z\}} c_{j, l(1)}\left[\delta_{\mathbf{r}_{0}} \widehat{p}_{l(1)}+\widehat{p}_{l(1)} \delta_{\mathbf{r}_{0}}\right], c_{l(1), l(2)}=(1 / 2 m) \delta_{l(1), l(2)}$. The aim of this appendix is to show that, for an Hamiltonian $H^{(n)}+V(\mathbf{r})$, the following form of the $j^{\text {th }}$ component of the probability current operator

$$
\begin{aligned}
\hat{J}_{j}^{(n)}\left(\mathbf{r}_{\mathbf{0}}\right)=\sum_{\substack{l(k) \in\{x, y, z\} \\
k=1, . ., n-1}} c_{j, l(1), \ldots, l(n-1)}\left[\delta_{\mathbf{r}_{0}} \widehat{p}_{l(1)} \widehat{p}_{l(2)} \ldots \widehat{p}_{l(n-1)}\right. & \\
& \left.+\widehat{p}_{l(1)} \delta_{\mathbf{r}_{0}} \widehat{p}_{l(2)} \ldots \widehat{p}_{l(n-1)}+\ldots+\widehat{p}_{l(1)} \widehat{p}_{l(2)} \ldots \widehat{p}_{l(n-1)} \delta_{\mathbf{r}_{0}}\right]
\end{aligned}
$$

gives back Eq. 14, The Dirac distribution interacts with the mixed powers of the current

operator so that the symmetrization procedure used in the construction of $\hat{J}_{j}^{(n)}\left(\mathbf{r}_{\mathbf{0}}\right)$ provides $(n-2)$ further summations with respect to $\widehat{J}_{j}^{(2)}\left(\mathbf{r}_{0}\right)$. The two definitions coincide only up to $n=2$. The extra terms are crucial in order to satisfy the continuity equation. We evaluate every term over a generic state $\psi$; for example the second term is of the shape

$$
\begin{aligned}
\left\langle\psi\left|\widehat{p}_{l(1)} \delta_{\mathbf{r}_{0}} \widehat{p}_{l(2)} \ldots \widehat{p}_{l(n-1)}\right| \psi\right\rangle & =\int \mathrm{d}^{3} r \psi^{*} \widehat{p}_{l(1)} \delta_{\mathbf{r}_{0}} \widehat{p}_{l(2)} \ldots \widehat{p}_{l(n-1)} \psi \\
& =\int \mathrm{d}^{3} r\left(\widehat{p}_{l(1)} \psi\right)^{\dagger} \delta_{\mathbf{r}_{0}} \widehat{p}_{l(2)} \ldots \widehat{p}_{l(n-1)} \psi \\
& =\left[\widehat{p}_{l(1)} \psi\left(\mathbf{r}_{0}\right)\right]^{\dagger} \widehat{p}_{l(2)} \ldots \widehat{p}_{l(n-1)} \psi\left(\mathbf{r}_{0}\right) .
\end{aligned}
$$


Then the $j^{\text {th }}$ Cartesian component of probability current for a generic state $J_{j}[\psi]$ can be written as:

$$
\begin{aligned}
& J_{j}^{(n)}[\psi]=\left\langle\psi\left|\hat{J}_{j}^{(n)}\left(\mathbf{r}_{\mathbf{0}}\right)\right| \psi\right\rangle=\sum_{\substack{l(k) \in\{x, y, z\} \\
k=1, ., n-1}} c_{j, l(1), \ldots, l(n)}\left[\psi^{\dagger} \widehat{p}_{l(1)} \ldots \widehat{p}_{l(n-1)} \psi+\ldots\right. \\
& \left.+\left(\widehat{p}_{l(1)} \ldots \widehat{p}_{l(k-1)} \psi\right)^{\dagger} \widehat{p}_{l(k)} \ldots \widehat{p}_{l(n-1)} \psi+\ldots+\left(\widehat{p}_{l(1)} \ldots \widehat{p}_{l(n-1)} \psi\right)^{\dagger} \psi\right]
\end{aligned}
$$

where $\psi=\psi\left(\mathbf{r}_{0}\right)$. From Eq. B3, we can find the generic divergence term related to the derivative with respect to $\widehat{p}_{j}$ :

$$
\begin{aligned}
& \widehat{p}_{j} J_{j}^{(n)}[\psi]=\sum_{\substack{l(k) \in\{x, y, z\} \\
k=1, . . n-1}} c_{j, l(1), \ldots, l(n)}\left[\psi^{\dagger} \widehat{p}_{j} \widehat{p}_{l(1)} \ldots \widehat{p}_{l(n-1)} \psi-\left(\widehat{p}_{j} \psi\right)^{\dagger} \widehat{p}_{l(1)} \ldots \widehat{p}_{l(n-1)} \psi\right. \\
& +\left(\widehat{p}_{l(1)} \ldots \widehat{p}_{l(k-1)} \psi\right)^{\dagger} \widehat{p}_{j} \widehat{p}_{l(k)} \ldots \widehat{p}_{l(n-1)} \psi-\left(\widehat{p}_{j} \widehat{p}_{l(1)} \ldots \widehat{p}_{l(k-1)} \psi\right)^{\dagger} \widehat{p}_{l(k)} \ldots \widehat{p}_{l(n-1)} \psi \\
& +\left(\widehat{p}_{l(1)} \ldots \widehat{p}_{l(k)} \psi\right)^{\dagger} \widehat{p}_{j} \widehat{p}_{l(k+1)} . . \widehat{p}_{l(n-1)} \psi-\left(\widehat{p}_{j} \widehat{p}_{l(1)} \ldots \widehat{p}_{l(k)} \psi\right)^{\dagger} \widehat{p}_{l(k+1)} \ldots \widehat{p}_{l(n-1)} \psi \\
& \left.+\ldots+\left(\widehat{p}_{l(1)} \ldots \widehat{p}_{l(n-1)} \psi\right)^{\dagger} \widehat{p}_{j} \psi-\left(\widehat{p}_{j} \widehat{p}_{l(1)} \ldots \widehat{p}_{l(n-1)} \psi\right)^{\dagger} \psi\right] .
\end{aligned}
$$

In Eq. B4 all the terms that have the same order in $k$ (two consecutive terms but the first one and the last one) vanish after summation over $j$ :

$$
\begin{aligned}
\sum_{j=\{x, y, z\}} \sum_{\substack{l(k) \in\{x, y, z\} \\
k=1, . . n-1}} c_{j, l(1), \ldots, c_{l(n)}\left[-\left(\widehat{p}_{j} \widehat{p}_{l(1)} \ldots \widehat{p}_{l(k-1)} \psi\right)^{\dagger} \widehat{p}_{l(k)} \ldots \widehat{p}_{l(n-1)} \psi\right.} \\
\left.+\left(\widehat{p}_{l(1)} \ldots \widehat{p}_{l(k)} \psi\right)^{\dagger} \widehat{p}_{j} \widehat{p}_{l(k+1)} . . \widehat{p}_{l(n-1)} \psi\right]=0
\end{aligned}
$$

Then the only terms still remaining in the summation are:

$$
\begin{aligned}
& \sum_{j=\{x, y, z\}} \widehat{p}_{j} J_{j}^{(n)}[\psi]=\widehat{\mathbf{p}} \cdot \mathbf{J}^{(n)}[\psi]
\end{aligned}
$$

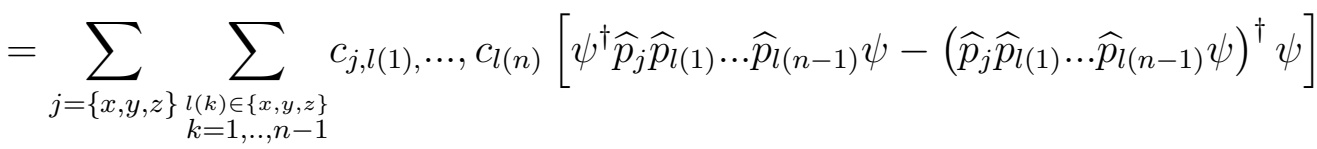

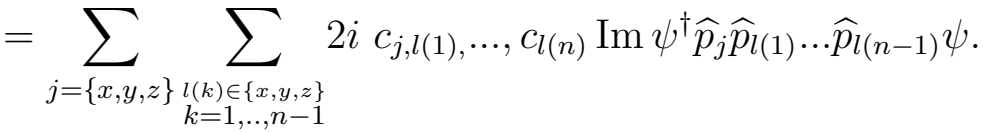

Now $\nabla \cdot \mathbf{J}^{(n)}[\psi]=(i / \hbar) \widehat{\mathbf{p}} \cdot \mathbf{J}^{(n)}[\psi]$ and Eq. B7 results in a collection of pure imaginary terms and the final expression for the divergence of the probability current reads:

$$
\nabla \cdot \mathbf{J}^{(n)}[\psi]=-\frac{2}{\hbar} \operatorname{Im} \sum_{j=\{x, y, z\}} \sum_{\substack{l(k) \in\{x, y, z\} \\ k=1, . ., n-1}} c_{j, l(1), \ldots, l(n)}\left(\psi\left|\widehat{p}_{j} \widehat{p}_{l(1)} \ldots \widehat{p}_{l(n-1)}\right| \psi\right) .
$$


Eventually

$$
\nabla \cdot \mathbf{J}[\psi]=\sum_{n} \nabla \cdot \mathbf{J}^{(n)}[\psi]
$$

* Electronic address: Henri-Jean.Drouhin@polytechnique.edu

1 C. Cohen-Tannoudji, B. Diu, and F. Laloë, Mecanique Quantique (Hermann, Paris, 1996), p. 238.

2 T. L. Hoai Nguyen, H.-J. Drouhin, J.-E. Wegrowe, and G. Fishman, Phys. Rev. B 79, 165204 (2009).

3 E. I. Rashba and V. I. Sheka, in Landau Level Spectroscopy, edited by G. Landwehr and E. I. Rashba (Elsevier, Amsterdam, 1991); p. 178.

4 M. D'yakonov and V. I. Perel', Zh. Eksp. Teor. Fiz. 60 , 1954 (1971) ; Sov. Phys. JETP 33, 1053 (1971).

5 E. I. Rashba, Phys. Rev. B 68, 241315(R) (2003).

6 J. Sinova, D. Culcer, Q. Niu, N. A. Sinitsyn, T. Jungwirth, and A. H. MacDonald Phys. Rev. Lett 92, 12 (2004).

7 E. B. Sonin, Phys. Rev. B 76, 033306 (2007) and Phys. Rev. B 77, 039901(E) (2008).

8 V. A. Sablikov and Y. Ya. Tkach, Phys. Rev. B 76, 245321 (2007).

9 V. I. Litvinov, Phys. Rev. B 82, 115321 (2010).

10 P. M. Haney and M. D. Stiles, Phys. Rev. Lett. 105, 126602 (2010).

11 Y. Li and R. Tao, Phys. Rev. B 75, 075319 (2007).

12 H.J. Drouhin, G. Fishman, and J.E. Wegrowe, Phys. Rev. B 83, 113307 (2011).

13 J. Shi, P. Zhang, D. Xiao, and Q. Niu, Phys. Rev. Lett. 96, 076604 (2006).

14 N. Sugimoto, S. Onoda, S. Murakami, and N. Nagaosa, Phys. Rev. B 73, 113305 (2006).

15 P. Zhang and Z. Wang, J. Shi, D. Xiao and Q. Niu, Phys. Rev. B 77, 075304 (2008).

16 A. Wong and F. Mireles, Phys. Rev. B 81, 085304 (2010).

17 J. Bendaniel and C.B. Duke, Phys. Rev. 152, 638 (1966).

18 W. Harrison, Phys. Rev. 123, 1 (1961).

19 A thermodynamic model only based on the continuity equation Eq. 5 cannot take into account the exchange of energy $e$ with the environment introduced by the current generator and the 
spin-orbit interaction. Indeed, the consequence of the Gibbs equation, $\mathrm{d} e / \mathrm{d} t=T \partial s / \partial t+\mu \partial \rho / \partial t$ (where $s$ is the entropy, $T$ the temperature, and $\mu$ is the chemical potential), is that the continuity equation of the density of carriers $\rho$ is necessarily determined by the power dissipated at the boundaries. At zero temperature, the source term is equal to the power dissipated divided by the chemical potential $\partial \rho / \partial t=(1 / \mu) \mathrm{d} e / \mathrm{d} t$. This is the reason why the decomposition between the divergence term and the source term $G$ cannot be unique without specifying the boundary conditions.

20 A. Messiah, Quantum Mechanics (North Holland Publishing Company, Amsterdam, 1962), Ch. XV, Sec. 2, p. 633.

21 Ref. 20, Ch. XV, Sec. 21, p. 675.

22 Ref. 20, Ch. X, Sec. 4, p. 372. 